\title{
Research on Human-Computer Interaction Scene Control Technology for Virtual Assembly of Aircraft
}

\author{
Han $\mathrm{Hu}^{1, \text { a }}$, Yong Wang ${ }^{1,2, \mathrm{~b},{ }^{*}}$ \\ ${ }^{1}$ School of Mechanical \& Vehicle Engineering, Linyi University, Linyi 276005, China \\ ${ }^{2}$ Shanghai Fire Research Institute of Ministry of Public Security, Shanghai 200438, China \\ ahanhu_lyu@126.com, bwangecust@163.com
}

Keywords: Aircraft, virtual assembly, control technology, simulation.

\begin{abstract}
For the problems of the unfairness of the human-computer interface in the process of traditional aircraft virtual assembly, the simulation results are not intuitive and the operator cannot directly observe the changes and adjustment of the parameters during the operation of the control system and so on. According to the actual control requirements of the aircraft virtual assembly process system, taking the human-computer interaction of 3D mouse as an example, this paper proposed a three-dimensional mouse viewpoint control method based on Open Inventor technology. Based on the operation mode and working principle of 3D mouse, a new human-computer interaction model the human-computer interaction based on Virtools is realized, the simulation scheme of the simulation object is established, the simulation scene is rendered, and the simulation process is controlled flexibly.
\end{abstract}

\section{Introduction}

Virtual assembly technology, as a high-end manufacturing and one of the important research content in the field of computer aided manufacturing, domestic and foreign scholars have been on the continuous research [1]. In aircraft design and manufacturing process involves many complicated steps, aircraft assembly is one of the crucial steps. Due to the structure size of aircraft is very larger, and complex shape, structures are mostly composed of sheet metal or composite wall panel thin shell structure, small junction surface, and the craft is more rigid, prone to deformation in the assembly process [2]. Although the domestic design and development phase of the aircraft, the basic realization of the three-dimensional digital intelligent design. However, in the aircraft assembly process design, the domestic is still the traditional two-dimensional way, computer-aided process design still stays at the two-dimensional level, which obviously cannot meet the three-dimensional digital conditions of aircraft assembly process design requirements.

However, the traditional aircraft virtual assembly man-machine interface is not friendly, virtual assembly real-time effect is not intuitive, the operator directly observe the control system in the process of running, the parameters of real-time changes, regulation and control is very difficult. Therefore, it is necessary to study the scene control technology of aircraft virtual assembly scene technology system in order to improve the effectiveness and controllability of design and experiment, improve the efficiency of research and development. According to the actual control requirements of the aircraft virtual assembly process system scenario, the human - computer interaction of 3D mouse is taken as an example to realize the human - computer interaction based on Virtools, the motion scheme of the simulation object is established, the simulation scene is rendered and the simulation process is controlled flexibly.

\section{Human-Computer Interaction Technology}

The so-called human-computer interaction refers to the use of computer input and output devices, in an efficient way to achieve the operator and the computer "dialogue" is an effective way to contact the operator and the machine, including the following two functions: (1)the output device to provide 
information to the operator, (2) the operator using the input device to transfer data information for the machine [3].

The introduction of human-computer interaction technology into the aircraft virtual assembly simulation process, which can provide a strong reference to optimize the assembly process design. Also, in the aircraft manufacturing process, virtual equipment, and human-computer interaction can provide technical support for the assembly of technical personnel.

\section{Human-Computer Interaction Based on 3D Mouse}

\subsection{D mouse technology.}

The 3D mouse is one of the true three-dimensional input devices, with a slight force applied to it above it, it can be monitored, can be decomposed into $\mathrm{X}, \mathrm{Y}, \mathrm{Z}$ three-axis translation and rotation around the three axes, so that the operation. Three-dimensional mouse translation direction and the working principle of the rotation axis diagram was shown in Figure 1.

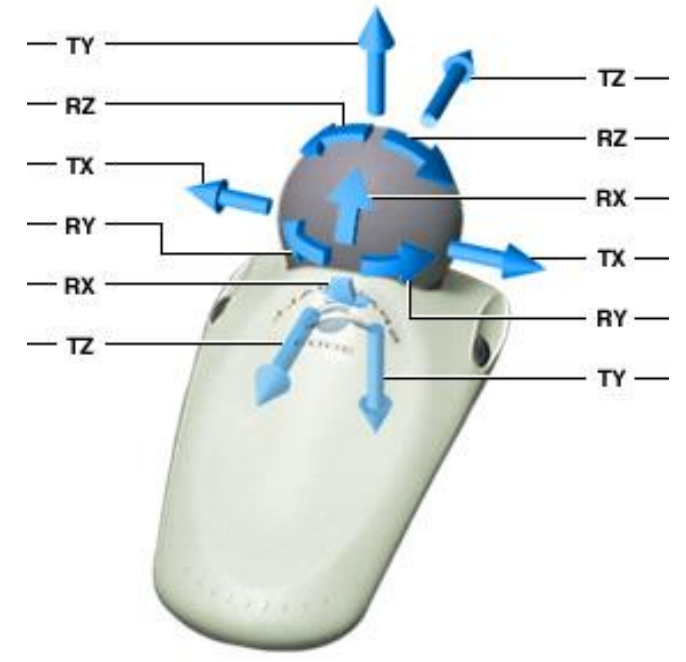

Fig. 1 The working principle of the translation direction and rotary shaft of the 3D mouse

Three-dimensional mouse ball is a hollow ball actually, the mouse ball "suspended" on the six leaf springs, the mouse outside the ball slot fixed in the spring of the spherical top. As long as the mouse ball by pulling or (and) to reverse, in order to eliminate the external force leaf spring need to shift accordingly, the force applied to the mouse ball is divided into six forces, light-emitting diode LED monitor according to light Sense principle will automatically measure the amount of each spring offset, and the detection of the mouse after the ball displacement.

\subsection{D mouse and virtual scene initialization.}

The three-dimensional mouse MFC message response machine is an effective means of communication, when an operational event is triggered, Windows obtains the event message and triggers the corresponding message response function. To implement this process, you must first register the 3D mouse message and create a message response function. The 3D mouse action event includes the control of the ball's operation and key operation, the former for the six-degree-of-freedom movement of the control point, which is used to set the motion mode, adjust the movement accuracy and start the control interface.

When the type of the event is to control the ball operation event, the orientation data of the 3D mouse output is obtained from the external interface, and the data is processed by the C3DMouse class, data; when the two coordinate systems are different, then the coordinate azimuth value is needed to correct the operation, and the processed data information is passed as a parameter to the response function that controls the value of the Open Inventor node. By changing the motion of the object in the virtual scene and Scene State and orientation, so as to achieve the purpose of controlling the state of view or assembly movement. 
In addition, the user in the use of three-dimensional mouse to browse the virtual assembly scene, you need to set a good point of view in advance, a scene for multiple observations. Therefore, the three-dimensional mouse human-computer interaction part gives the "view teaching", "save browsing path" and "automatic browsing" and other functions. Table 1 is the viewpoint storage structure and its initial value.

Table 1 The Viewpoint storage structure and its initial value

\begin{tabular}{cc}
\hline Project & Initial value \\
\hline Point space coordinates & viewpoint_x, viewpoint_y, viewpoint_z \\
Perspective angle variable & viewpoint_anglex=viewpoint_anglez=viewpoint_angley=0 \\
$\begin{array}{c}\text { Foreground object spatial coordinates } \\
\text { Foreground object rotation angle }\end{array}$ & $\mathrm{m} \_\mathrm{x}=\mathrm{m} \_\mathrm{y}=\mathrm{m} \_\mathrm{z}=0$ \\
$\begin{array}{c}\text { Followed by the ViewPointStructure } \\
\text { pointer }\end{array}$ & m_angleX=m_angleY=m_angleZ=0 \\
& nextViewPoint $=\mathrm{NULL}$ \\
\hline
\end{tabular}

\section{Applications}

In the test test analysis process, take the aircraft assembly outline as an example. Using the keyboard and mouse to control the scene, to achieve the function of human-computer interaction, such as the mouse with the left side of the $\mathrm{ABC}$ click on the side of the program number can start the corresponding scene simulation and operation of the text description, the keyboard keys "1" "Corresponds to steps 010 to 090. In addition, the "z" key causes the animation to stop, the space bar causes the animation to pause, "w", "s", "a", "d" four keys to control the top, bottom, left and right of the lens, - "two keys to control the lens zoom. The experimental results are shown in Figure 2.

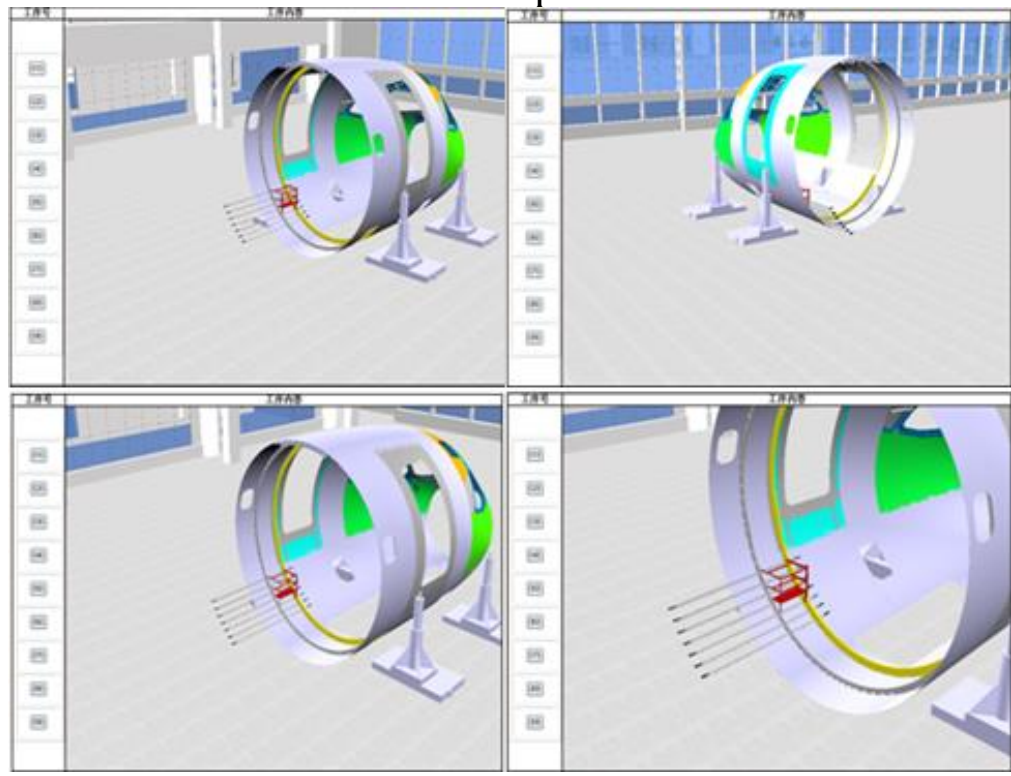

Fig. 2 The assembly outline of aircraft

\section{Summary}

(1) The three-dimensional mouse human-computer interaction technology, including: human-computer interaction technology, three-dimensional mouse technology, three-dimensional mouse super-sensing device principle and three-dimensional mouse point of view control methods were introduced analysis.

(2) According to the actual control requirements of the aircraft virtual assembly process system scene, the human-computer interaction based on Virtools is realized by taking the human-computer interaction of 3D mouse as an example.

(3) This paper presents a method based on Open Inventor 3D mouse view control technology. A new human-computer interaction model is built by using the operation principle of 3D mouse, and the 
multi-angle observation of the simulation process of aircraft assembly process in the virtual scene is realized.

\section{Acknowledgements}

Funding: This work was supported by "National key R \& D plan of China" [2016YFC0800607].

\section{References}

[1]. Q.Yang, D.L.Wu. Assembly operation process planning by mapping a virtual assembly simulation to real operation. Computers in Industry. Vol. 64 (2013) No. 7, p. 869-879.

[2]. W.J.Wu: Research and Application of Virtual Assembly Technology Based on DELMIA (Doctor, Nanjing University of Aeronautics and Astronautics, China, 2008).P.5-16.

[3]. D.Q. Nguyen, R. Fedkiw, H.W. Jensen. Physically based modeling and animation of fire. Proceedings of the 29th annual conference on Computer graphics and interactive techniques. New York, USA, 2002, p. 721-728. 